\title{
Drosophila Aurora-A kinase inhibits neuroblast self-renewal by regulating aPKC/Numb cortical polarity and spindle orientation
}

\author{
Cheng-Yu Lee, ${ }^{1,3,4}$ Ryan O. Andersen, ${ }^{1,3}$ Clemens Cabernard, ${ }^{1}$ Laurina Manning, ${ }^{1}$ Khoa D. Tran, ${ }^{1}$ \\ Marcus J. Lanskey, ${ }^{1}$ Arash Bashirullah, ${ }^{2}$ and Chris Q. Doe ${ }^{1,5}$ \\ ${ }^{1}$ Institutes of Neuroscience and Molecular Biology, Howard Hughes Medical Institute, University of Oregon, Eugene, \\ Oregon 97403, USA; ${ }^{2}$ Department of Human Genetics, Howard Hughes Medical Institute, University of Utah School of \\ Medicine, Salt Lake City, Utah 84112, USA
}

\begin{abstract}
Regulation of stem cell self-renewal versus differentiation is critical for embryonic development and adult tissue homeostasis. Drosophila larval neuroblasts divide asymmetrically to self-renew, and are a model system for studying stem cell self-renewal. Here we identify three mutations showing increased brain neuroblast numbers that map to the aurora- $A$ gene, which encodes a conserved kinase implicated in human cancer. Clonal analysis and time-lapse imaging in aurora- $A$ mutants show single neuroblasts generate multiple neuroblasts (ectopic self-renewal). This phenotype is due to two independent neuroblast defects: abnormal atypical protein kinase $\mathrm{C}(\mathrm{aPKC}) / \mathrm{Numb}$ cortical polarity and failure to align the mitotic spindle with the cortical polarity axis. numb mutant clones have ectopic neuroblasts, and Numb overexpression partially suppresses aurora- $A$ neuroblast overgrowth (but not spindle misalignment). Conversely, mutations that disrupt spindle alignment but not cortical polarity have increased neuroblasts. We conclude that Aurora-A and Numb are novel inhibitors of neuroblast self-renewal and that spindle orientation regulates neuroblast self-renewal.
\end{abstract}

[Keywords: Aurora-A; Drosophila; neuroblast; numb; self-renewal; spindle orientation]

Supplemental material is available at http://www.genesdev.org.

Received September 1, 2006; revised version accepted November 3, 2006.

The precise regulation of stem cell self-renewal versus differentiation is critical for normal development, tissue homeostasis, and regeneration. The stem cell pool can be expanded via symmetric cell division or can be kept at a steady state while generating differentiated progeny via asymmetric cell division (Morrison and Kimble 2006). Drosophila larval brain neuroblasts have many stem cell attributes-such as the ability to remain proliferative, undifferentiated, and nontumorous for a hundred or more cell divisions while generating post-mitotic neurons and glia-and thus they have recently served as a paradigm for studies of self-renewal versus differentiation (Betschinger et al. 2006; Lee et al. 2006a,b). Neuroblasts divide asymmetrically to self-renew and to generate differentiating ganglion mother cells (GMCs), which typically generate two post-mitotic neurons or glia.

\footnotetext{
${ }^{3}$ These authors contributed equally to this work.

${ }^{4}$ Present address: Center for Stem Cell Biology, Life Sciences Institute, University of Michigan, Ann Arbor, MI 48109, USA.

${ }^{5}$ Corresponding author.

E-MAIL cdoe@uoneuro.uoregon.edu; FAX (541) 346-4736.

Article is online at http://www.genesdev.org/cgi/doi/10.1101/gad.1489406.
}

Neuroblast self-renewal requires establishment and maintenance of proper apical/basal cortical polarity. Two protein complexes are localized to the neuroblast apical cortex and partitioned into the neuroblast during asymmetric division: the Par complex and the Pins complex. The evolutionarily conserved Par complex contains Bazooka (Baz; Par-3 in mammals)/Par-6/atypical protein kinase $\mathrm{C}(\mathrm{aPKC})$ proteins, and the Pins complex contains Partner of Inscuteable (Pins)/G $\alpha$ i proteins; these complexes are linked by the Inscuteable (Insc) protein, which binds both Baz and Pins (Cai et al. 2003; Fuse et al. 2003; Yu et al. 2003; Siegrist and Doe 2005). Proteins localized to the basal cortex and partitioned into the GMC include Numb, Miranda (Mira), Brain tumor (Brat), and Prospero (Pros) (for review, see Betschinger and Knoblich 2004). Mislocalization of aPKC to the basal (GMC) cortex triggers ectopic neuroblast self-renewal, resulting in a dramatic expansion of the neuroblast population (Lee et al. 2006a), whereas depletion of Pros or Brat from the GMC also leads to GMC-neuroblast transformation and overproduction of neuroblasts (Bello et al. 2006; Betschinger et al. 2006; Lee et al. 2006b). Thus, establishing proper 
cortical polarity is essential for neuroblast self-renewal and GMC differentiation.

A second, more speculative pathway for regulating neuroblast self-renewal involves spindle orientation. In theory, if the neuroblast mitotic spindle aligns orthogonal to the apical/basal polarity axis, both neuroblast progeny would inherit apical and basal proteins, and both might assume a neuroblast identity (similar to an epithelial cell division, which partitions apical and basolateral membrane domains equally into both siblings). The linkage between spindle and apical cortex is provided, in part, by the Mud protein (Mushroom body defective; the Drosophila NuMA ortholog), which directly binds both microtubules and cortical Pins protein; mud mutants show misalignment of the mitotic spindle without altering cortical polarity (Bowman et al. 2006; Izumi et al. 2006; Siller et al. 2006). mud mutant brains have an increase in neuroblast number (Prokop and Technau 1994; Bowman et al. 2006), and it is tempting to speculate that the symmetric divisions lead to a pair of sibling neuroblasts and thus to the observed increase in neuroblast number, but this has not been confirmed by clonal analysis.

Here we describe the results of a genetic screen for additional regulators of neuroblast self-renewal. We identify three mutants showing a massive increase in larval neuroblast numbers, without any detectable disruption of optic lobe or imaginal disc epithelia, and all are alleles of the evolutionarily conserved mitotic kinase aurora-A (aurA). Mammalian AurA kinase is localized to centrosomes and is considered an oncogene (for review, see Giet et al. 2005). In Drosophila, AurA is required for centrosome maturation, cell cycle progression, Numb protein localization during sense organ precursor asymmetric cell division, and astral microtubule length in S2 cells and larval neuroblasts (Glover et al. 1995; Berdnik and Knoblich 2002; Giet et al. 2002). Here we show that loss of AurA leads to neuroblast brain tumors, which arise primarily due to defects in Numb localization and secondarily due to defects in spindle-to-cortical polarity alignment. Our data define a role for Numb in the GMC as an inhibitor of neuroblast self-renewal, and provide direct evidence that spindle orientation can regulate neuroblast self-renewal.

\section{Results}

aurora-A mutants have too many larval neuroblasts

To identify genes that regulate neuroblast self-renewal, we screened a collection of pupal lethal mutants ( $\mathrm{L}$. Wang, J. Evans, H. Andrews, R. Beckstead, C.S. Thummel, and A. Bashirullah, in prep.) for changes in central brain neuroblast number. Three mutants [1(3)LL-8839, 1(3)LL-14641, 1(3)LL-17961] had a massive increase in larval neuroblasts, developing $>1000$ central brain neuroblasts at stages where wild-type larvae have only 95100 neuroblasts; in addition, the mutant neuroblasts were maintained into pupal stages, whereas wild-type pupae have few neuroblasts (Fig. 1A-C). The mutants had normal optic lobe and imaginal disc epithelial morphology (Fig. 1B'; data not shown), unlike previously identified tumor suppressor mutants (for review, see Hariharan and Bilder 2006). The mutations were deficiency and complementation mapped to the aurA locus, and the aurA $A^{87 a c-3}$ mutant (Glover et al. 1995) showed a similar supernumerary brain neuroblast phenotype (data not shown). Sequencing of the mutant alleles revealed that 1(3)LL-14641 has a single base change resulting in a $\mathrm{V} \rightarrow \mathrm{E}$ change at position 302 , within the kinase activation loop (Cheetham et al. 2002); 1(3)LL-17961 has a single base change resulting in a $\mathrm{D} \rightarrow \mathrm{N}$ change at position 334 that is predicted to destabilize $\alpha$-helical packing; and 1(3)LL-8839 has a single base change resulting in a $\mathrm{K} \rightarrow$ stop change at position 377 that deletes the $\mathrm{C}$ terminus of the protein (Fig. 1D); thus these are new aurA alleles. The AurA kinase is detected in the cytoplasm and on centrosomes in neuroblasts and sense organ precursors (Berdnik and Knoblich 2002), as is an AurA:GFP fusion protein expressed in neuroblasts (Supplementary Fig. 1; data not shown), consistent with a role in neuroblast asymmetric cell division.

We next asked if the ectopic neuroblasts in aurA mutants had all the properties of wild-type neuroblasts. In wild-type brains, neuroblasts express the markers Deadpan (Dpn), Mira, Worniu (Wor); fail to express the neuronal differentiation markers nuclear Pros and Elav; and are proliferative based on their ability to incorporate BrdU (Fig. 1E). In aurA mutants, all ectopic neuroblasts have the same properties (Fig. $1 F$; data not shown). In addition, we used BrdU pulse/chase experiments to determine if aurA neuroblasts could generate neurons. Immediately after a BrdU pulse, both wild-type and aurA mutant neuroblasts-but no neurons-incorporated BrdU (Fig. 1G,I); following a 24-h BrdU-free chase, all BrdU labeling was detected exclusively in the Elav ${ }^{+}$neurons (Fig. 1H,J). Thus, the ectopic neuroblasts in aurA mutant larvae show normal marker expression and proliferation, and are able to generate post-mitotic neurons.

\section{Single aurora-A mutant neuroblasts generate clones containing multiple neuroblasts}

To determine the origin of the extra neuroblasts in the mutant brains, we generated positively marked clones in single neuroblasts within aurA mutant brains, at a low frequency of one to two per brain lobe to keep each clone well separated. In wild type, genetically marked $\mathrm{\beta gal}^{+}$ clones induced in a single neuroblast always mark one large neuroblast and a population of smaller GMC/neuronal progeny (Fig. $1 \mathrm{~K})$. In contrast, single neuroblast clones induced in aurA mutants typically contained multiple neuroblasts as well as a pool of smaller GMC/ neuronal progeny (Fig. 1L). Thus, aurA mutant neuroblasts undergo occasional divisions in which both siblings assume the neuroblast fate, leading to an expansion of the neuroblast population (Fig. 1M). Importantly, neuroblast-specific expression of AurA:GFP (wor-gal4 UASaurA:GFP) is able to significantly rescue the aurA super- 
Lee et al.
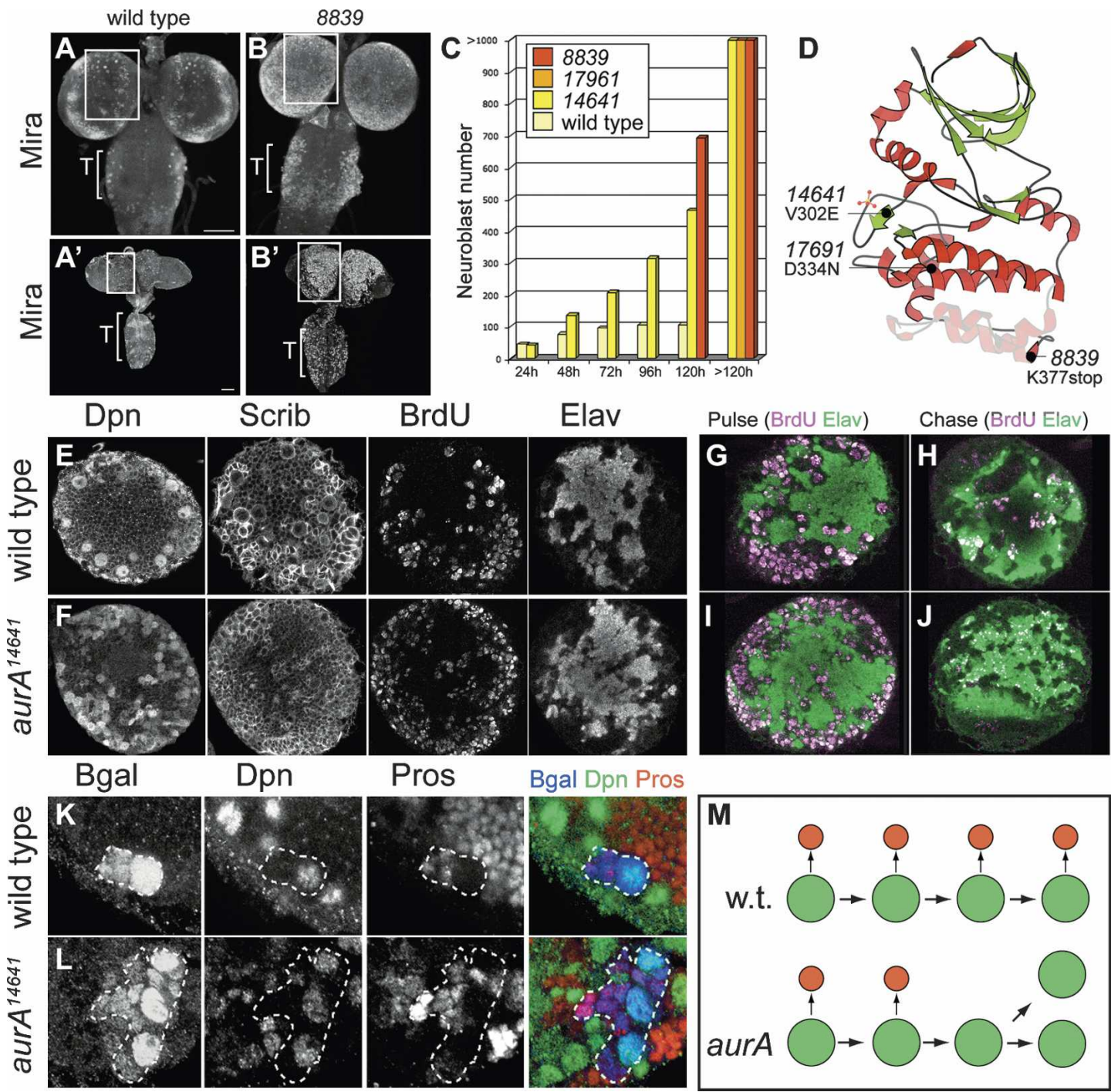
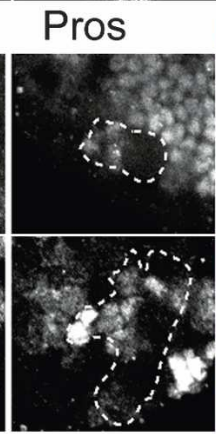

Bgal Dpn Pros

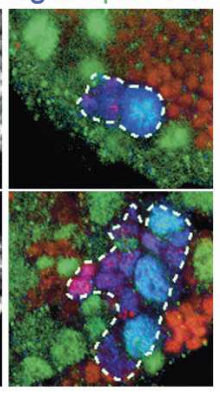

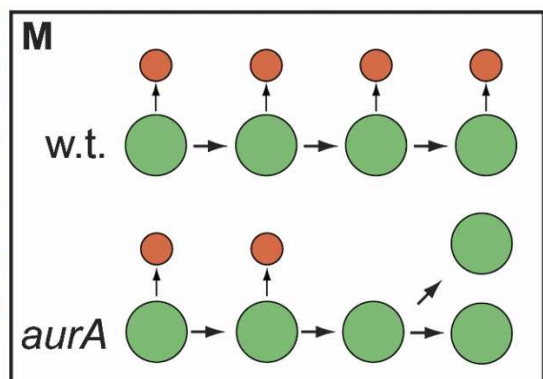

Figure 1. aurora-A mutants have too many larval neuroblasts. $(A, B)$ Larval brains at $96 \mathrm{~h}$ ALH showing increased Mira ${ }^{+}$neuroblasts in the mutant central brain (boxed) and thorax $(\mathrm{T}) \cdot\left(\mathrm{A}^{\prime}, \mathrm{B}^{\prime}\right)$ Pupal brains at 12-18 $\mathrm{h}$ after pupariation showing increased numbers of proliferative $\mathrm{Dpn}^{+}$neuroblasts and expanded CNS size. (C) Quantification of brain neuroblast numbers (using Dpn or Mira markers). $(D)$ Molecular lesions in aurA alleles superimposed on the crystal structure of human Aurora-A (Cheetham et al. 2002; Nowakowski et al. 2002). Lightly shaded region is absent in $\operatorname{aur}^{8839} \cdot(E, F)$ aurA mutant neuroblasts are correctly specified. Wild-type $(E)$ and $\operatorname{aur}^{14641}(F)$ neuroblasts are $\mathrm{Dpn}^{+}$Elav $^{-}$and incorporate BrdU, showing that they are specified correctly and are proliferative. (G-J) aurA mutant neuroblasts can proliferate and generate neuronal progeny. Wild-type $(G, H)$ and aurA ${ }^{14641}(I, J)$ 96-h-ALH larvae incorporate BrdU into neuroblasts following a 4-h BrdU pulse $(G, I)$, and "chase" the BrdU into post-mitotic neurons following a 24-h BrdU-free chase $(H, J) .(K-M)$ aurA mutant neuroblasts generate multiple neuroblasts. Single-neuroblast clones identified by $\beta$ gal expression (circled with the dotted line). $(K)$ In wild type, clones contain one $\mathrm{Dpn}^{+}$neuroblast and many nuclear Pros ${ }^{+}$progeny. $(L)$ In aur $A^{14641}$, clones contain multiple Dpn ${ }^{+}$neuroblasts and nuclear Pros ${ }^{+}$progeny. (M) Summary. (Green) Neuroblasts; (red) GMCs.

numerary neuroblast phenotype, showing that the phenotype is due to loss of AurA within neuroblast lineages (Supplementary Fig. 2).We conclude that the evolutionarily conserved AurA kinase is required to inhibit neuroblast self-renewal and promote GMC/neuronal differentiation.

\section{Aurora-A regulates aPKC and Numb cortical polarity}

In wild-type neuroblasts, Baz/Par6/aPKC and Insc/Pins/ Goi proteins are localized to the apical cortex of meta- phase neuroblasts (Fig. 2A-C; data not shown). In aurA mutants, we find that aPKC can be uniform cortical or show ectopic cortical patches; all other apical proteins assayed show normal localization (Fig. 2D-G; data not shown). Ectopic aPKC is observed in a small fraction of neuroblasts at early larval stages $(48-72 \mathrm{~h}$ after larval hatching $[\mathrm{ALH}])$ and becomes much more common at later stages when the increase in neuroblast number is the most dramatic (96-120 h ALH). aPKC; aurA double mutants completely suppress ectopic neuroblast formation (Supplementary Fig. 2), showing that aPKC is re- 


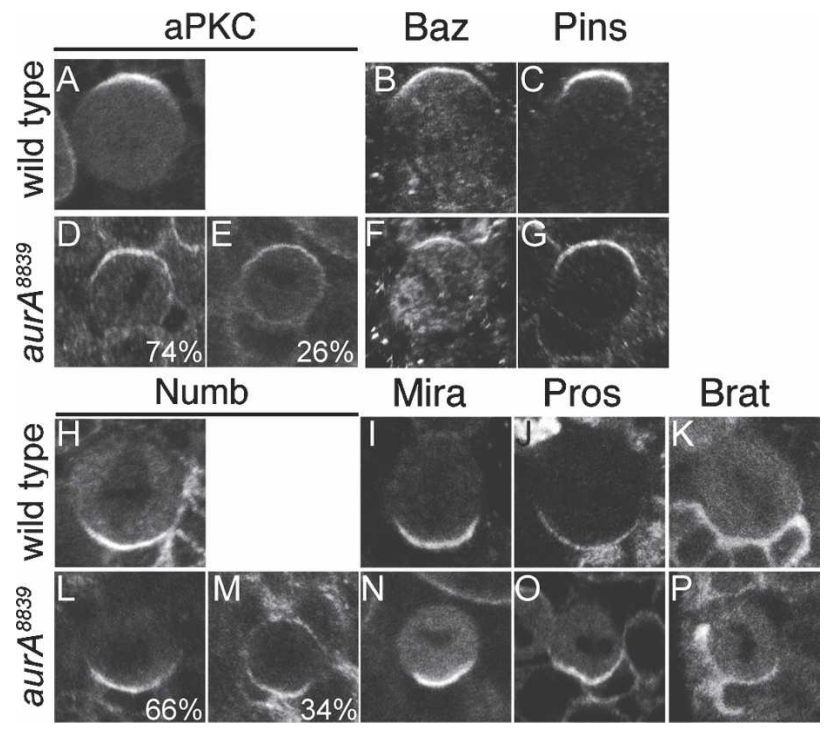

Figure 2. aurora- $A$ neuroblasts have ectopic aPKC and delocalized Numb. $(A-C, H-K)$ Wild-type mitotic neuroblasts at 120 $\mathrm{h}$ ALH show normal apical protein localization $(A-C)$ and basal protein localization $(H-K)$. $(D-G, L-P)$ aur $A^{8839}$ mutant mitotic neuroblasts at $120 \mathrm{~h}$ ALH show delocalization of aPKC (26\%, $n=47)$ and Numb (34\%, $n=69)$. Baz, Pins, Mira, Pros, Brat $(B, C, I-K)$ and Gai, Insc, and Pon (data not shown) have essentially normal localization.

quired for the aurA supernumerary neuroblast phenotype. This rescue is specific (e.g., not simply due to aPKC mutants arresting the neuroblast cell cycle or inducing neuroblast cell death) because $a P K C$ mutants can only weakly suppress the brat mutant supernumerary neuroblast phenotype (Lee et al. 2006b). We conclude that aurA mutants disrupt aPKC but not other tested apical polarity proteins; that aPKC delocalization coincides with the increase in neuroblast numbers; and that aPKC is required for the ectopic neuroblasts observed in aurA mutants.

Loss of the basal proteins Brat or Pros can generate supernumerary neuroblast phenotypes (Bello et al. 2006; Betschinger et al. 2006; Lee et al. 2006b), so we tested whether aurA mutants have defects in basal protein localization. Wild-type neuroblasts localize Numb, Miranda, Pros, and Brat to the basal cortex at metaphase (Fig. 2H-K). In aurA mutants, we observed normal Mira/ Pros/Brat protein basal crescents at metaphase (Fig. 2I$\mathrm{K})$, but Numb was either relatively normal (Fig. 2L) or delocalized around the cortex (Fig. 2M). This is similar to the Numb localization defects in aurA mutant mitotic sense organ precursors (Berdnik and Knoblich 2002; see below).

\section{Numb inhibits neuroblast self-renewal}

The role of Numb during larval neuroblast asymmetric division has never been investigated, so we assayed the phenotype of numb mutant clones generated in single larval neuroblasts. Wild-type clones always show one neuroblast and its family of neuronal progeny (Fig. 3A), whereas numb mutant clones always contain multiple neuroblasts plus neuronal progeny (Fig. 3B). Thus, the numb clones are similar to the aurA mutant brains in their ability to expand the neuroblast population while still producing differentiated $\mathrm{Pros}^{+} \mathrm{Elav}^{+}$neurons. Interestingly, the numb and AurA mutant phenotypes are distinct from mira, pros, or brat mutant single neuroblast clones, which contain few or no differentiated neurons (Supplementary Fig. 3). We conclude that AurA is required to restrict Numb to the basal cortex and that Numb inhibits neuroblast selfrenewal.

We next tested whether Numb acts downstream from AurA by using the neuroblast-specific wor-Gal4 line to overexpress Numb in aurA mutant neuroblasts and GMCs. While aur $A^{14641}$ mutants have $>450$ neuroblasts per brain lobe, overexpression of Numb is sufficient to partially rescue the supernumerary neuroblast phenotype in aur $A^{14641}$ mutants $(\sim 175$ neuroblasts per lobe) at the same larval stage (Fig. 3C-E); this is similar to the number of neuroblasts seen in mutants that affect spindle orientation but not cortical polarity such as cnn (Fig. 3C) or mud (Prokop and Technau 1994; Bowman et al. 2006). Thus, Numb overexpression appears to suppress the cortical polarity component of the aurA supernumerary neuroblast phenotype. We conclude that Numb acts downstream from AurA to inhibit neuroblast self-renewal.

\section{Aurora-A regulates alignment of the mitotic spindle with the cortical polarity axis}

We next tested whether spindle orientation defects could contribute to the production of ectopic neuroblasts in aur $A$ mutants. In wild-type metaphase neuroblasts, the mitotic spindle is aligned within $15^{\circ}$ of the center of the apical/basal cortical polarity axis (Siller et al. 2005, 2006; Bowman et al. 2006). In aurA metaphase neuroblasts, the mitotic spindle is essentially randomized relative to the apical/basal cortical polarity axis (Fig. 4B,C). Because some aurA neuroblasts have multiple centrosomes (wild type: $2.0, n=25$; aurA: $3.8, n=45$ ) and multipolar spindles, we only scored neuroblasts where a clear bipolar spindle could be observed. In addition, aur $A$ mutant neuroblasts have defects in telophase spindle orientation, with $\sim 15 \%$ of the neuroblasts dividing symmetrically (Fig. 4D,F; see below) - a phenotype that is never observed in wild-type neuroblasts (Fig. 4E). Spindle orientation defects are not due to loss of cortical Mud protein (which is essential for proper spindle orientation) (Bowman et al. 2006; Izumi et al. 2006; Siller et al. 2006), because Mud remains cortical in aurA mutant neuroblasts (Fig. 4G,H). Interestingly, overexpression of Numb does not rescue the aurA spindle orientation defect (Fig. 4D), consistent with AurA regulating cell polarity and spindle orientation phenotypes via two distinct pathways (see Discussion). We conclude that AurA is essential for proper alignment of the mitotic spindle to the neuroblast cortical polarity axis. 
Lee et al.

Figure 3. Numb acts downstream from AurA to inhibit neuroblast self-renewal. $(A, B)$ Numb inhibits neuroblast self-renewal. $(A)$ Wild-type neuroblast clones always contain a single neuroblast and multiple differentiated progeny. (B) numb $b^{2}$ mutant clones contain multiple neuroblasts. Clones were induced by standard methods at $0-24 \mathrm{~h} \mathrm{ALH}$ and processed at $72 \mathrm{~h} \mathrm{ALH} \mathrm{for} \mathrm{mCD8} \mathrm{(clone} \mathrm{marker),}$ Mira (neuroblast marker), and Pros (GMC/ neuron marker). (C-E) Numb overexpression can suppress supernumerary neuroblast formation in aurA mutants. (C) Quantification of $\mathrm{Mira}^{+}$neuroblast numbers for a $120-\mathrm{h}-\mathrm{ALH}$ brain lobe of the indicated genotypes. $(D, E)$ Single optical section $8 \mu \mathrm{m}$ below the dorsal surface of a 120-h-ALH brain lobe stained for the Mira neuroblast marker in $\operatorname{aur}^{14641}(D)$ or aurA $A^{14641}$ wor-gal4 UAS-numb (E) larvae.

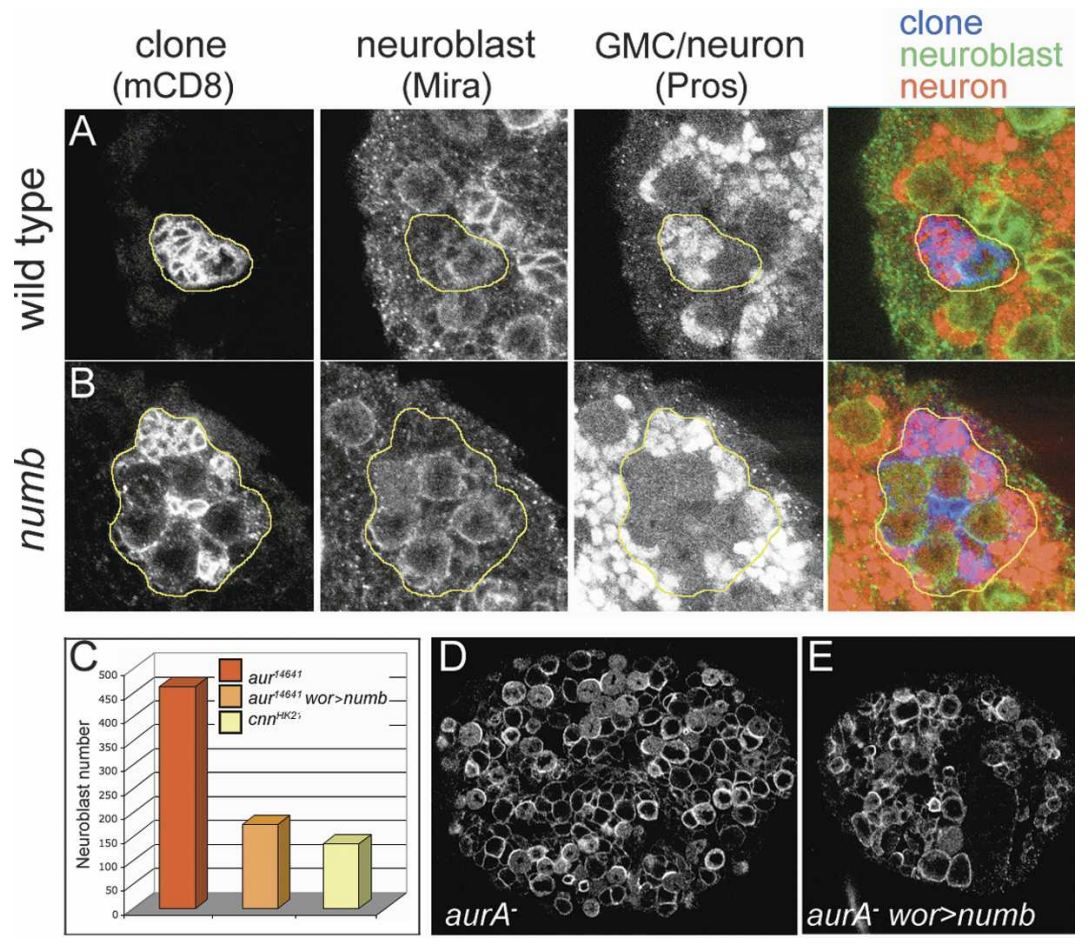

To determine if the aurA cell polarity and spindle orientation phenotypes are specific to neuroblasts, we also assayed asymmetric cell division of the pupal external sensory organ precursor (SOP). In wild-type pupae, the SOP localizes Numb and Pins to the anterior cortex and $\mathrm{Baz} / \mathrm{Par} 6 / \mathrm{aPKC}$ to the posterior cortex, and the mitotic spindle is tightly aligned with the anterior/posterior cortical polarity axis (Fig. 4I,J; for review, see Betschinger and Knoblich 2004). In addition, we find that the spindle anchoring protein Mud is centrosomal and enriched at the anterior and posterior cortex (Fig. 4K). In aurA mutant SOPs, cortical polarity is established normally based on proper anterior Pins crescent formation, but Numb is delocalized (confirming previous findings; Berdnik and Knoblich 2002). Importantly, we observe nearly random spindle orientation relative to the SOP cortical polarity axis (Fig. 4L-N), similar to the neuroblast spindle orientation phenotype. The most frequent spindle orientation is along the proximodistal axis, perhaps reflecting a secondary spindle orientation cue from the planar cell polarity system. We conclude that AurA is required in both neuroblasts and SOPs to align the mitotic spindle with the cortical polarity axis.

\section{aurora-A neuroblasts divide symmetrically to expand the neuroblast population}

It has been proposed that physically symmetric cell divisions can lead to expansion of the larval neuroblast population during mutant or wild-type insect development (Nordlander and Edwards 1970; Farris et al. 1999; Bowman et al. 2006; Siller et al. 2006), but this attractive hypothesis has never been directly tested. Here we use time-lapse imaging to assay the frequency and cell fate consequences of neuroblast symmetric divisions in aurA ${ }^{14641}$ mutants. We imaged histone:GFP (HisAvD:GFP) in explanted larval brains; these wholebrain cultures are healthy enough to allow us to follow $>10$ wild-type neuroblast cell divisions (see Materials and Methods). We assayed division symmetry by measuring both sibling nuclear size (with histone:GFP) and sibling cell size (using background fluorescence signal). We found that wild-type neuroblasts always divide asymmetrically with regard to sibling cell and nuclear size $(100 \%, n=11)$ (Fig. 5A,D; Supplementary Movie 1). In contrast, aur $A$ mutant neuroblasts show both asymmetric cell divisions (Fig. 5B,D; Supplementary Movie 2) and symmetric cell divisions $(17 \%, n=42)$ (Fig. 5C,D; Supplementary Movies 3, 4). Thus, aurA ${ }^{14641}$ neuroblasts show a significant number of symmetric divisions; this could contribute to the supernumerary neuroblast phenotype if both progeny retained a neuroblast identity. In support of this model, we observed a neuroblast lineage tree containing two symmetric divisions followed by an asymmetric cell division (Fig. $5 \mathrm{E}$ ). This shows that a symmetric neuroblast division can produce additional neuroblasts with the potential for asymmetric cell division-a hallmark of neuroblast identity—and suggests that symmetric divisions can expand the neuroblast population.

Reduction in mammalian AurA kinase levels results in mitotic delay (Dutertre et al. 2002; Du and Hannon 2004). Time-lapse analysis of aurA mutant neuroblasts revealed delays in prometaphase and metaphase (Fig. 5F) as well as increased overall cell cycle length (data not shown). In addition, we observe an increased mitotic in- 

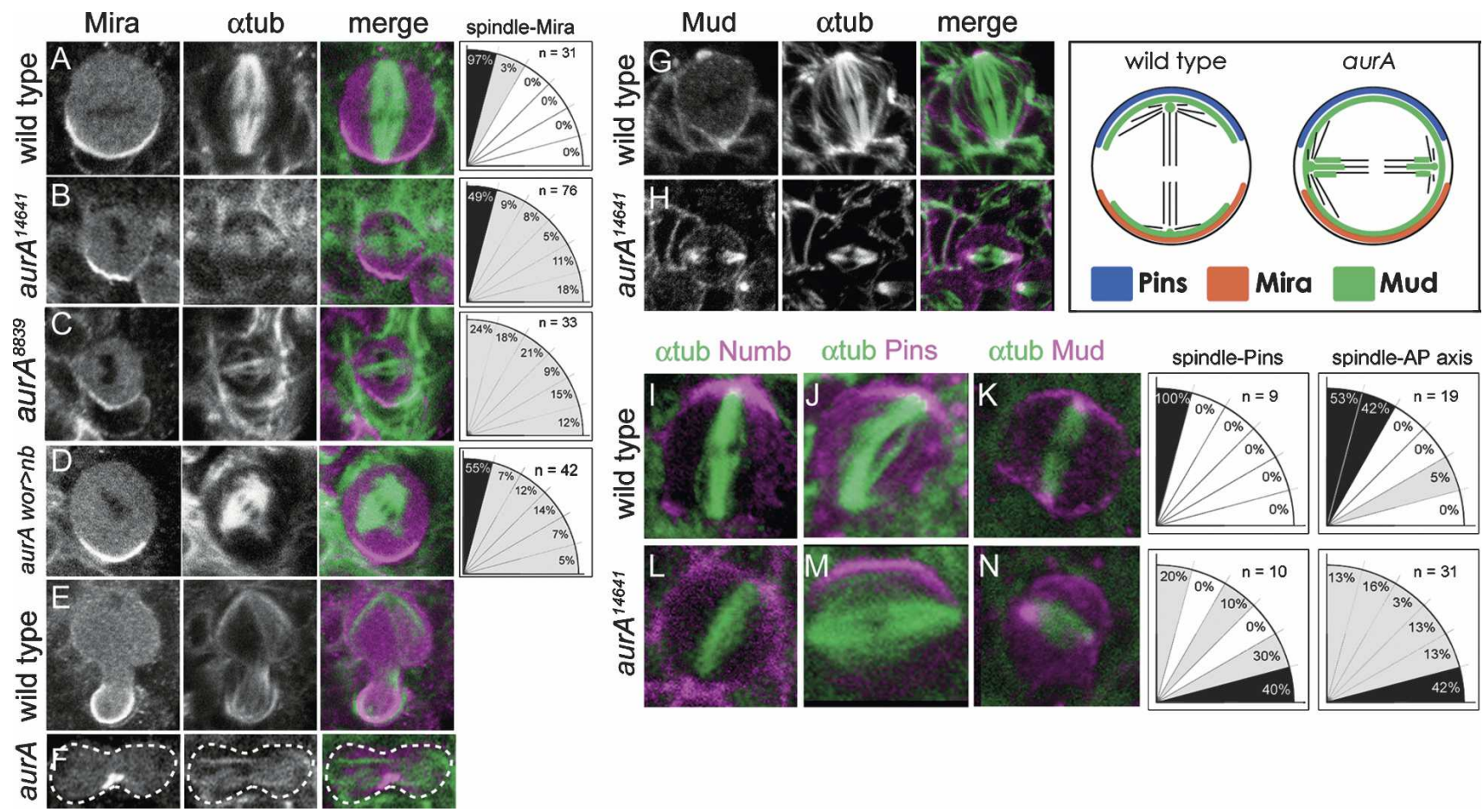

Figure 4. aurora- $A$ neuroblasts and SOPs fail to align the mitotic spindle with the cortical polarity axis. $(A-F)$ aur $A$ mutant neuroblasts fail to align the mitotic spindle with the cortical polarity axis. $(A)$ Wild-type metaphase $(A)$ or telophase $(E)$ 120-h-ALH neuroblasts showing tight alignment of the mitotic spindle ( $\alpha$ tub, $\alpha$-tubulin) with the apical/basal cortical polarity axis (Mira, Miranda basal marker). $(B, C, F)$ aur $A^{8839}$ mutant larval metaphase $(B, C)$ or telophase $(F)$ 120-h-ALH neuroblasts showing misalignment of the mitotic spindle relative to the apical/basal cortical polarity axis. Quantification of metaphase spindle orientation is shown at right (black, $>40 \%$ within the $15^{\circ}$ sector; gray, $1 \%-39 \%$; white, $\left.0 \%\right) .(G, H)$ The Mud spindle anchoring protein is cortical and centrosomal in both wild-type $(G)$ and aur $A^{14641}(H)$ metaphase neuroblasts, although aur $A^{14641}$ mutants have increased Mud staining on the spindle. (Right panel) Summary. (I,J) Wild-type and aurA $A^{14641}$ mutant mitotic SOPs at 16-20 h APF; anterior up, labeled for the indicated cortical polarity marker (magenta) and the $\alpha$-tubulin spindle marker (green). The spindle is often orthogonal to the cortical polarity axis. Quantification of metaphase spindle orientation is shown at right.

dex (wild type: $7.6 \%$ prometaphase-metaphase, $n=690$; aurA: $14.2 \%$ prometaphase-metaphase; $n=2753$ ) in fixed preparations. It is formally possible that the cell cycle delays are the cause of the neuroblast cell polarity and supernumerary neuroblast phenotype (Fichelson and Gho 2004). However, we have previously shown that metaphase-arrested neuroblasts have normal cortical polarity, and that Lis1 and Glued mutants have cell cycle delays without a matching neuroblast cortical polarity or supernumerary neuroblast phenotype (Spana and Doe 1995; Broadus and Doe 1997; Siller et al. 2005). We conclude that aurA mutant neuroblasts show cell cycle delays, but that these delays do not prevent or cause the observed increase in brain neuroblasts.

\section{Discussion}

We have shown that mutations in aurA lead to a massive increase in larval brain neuroblasts. The major cause of this phenotype appears to be misregulation of neuroblast cortical polarity. One cortical polarity defect is increased basal localization of aPKC, which we previously showed was sufficient to induce ectopic neuroblasts (Lee et al. 2006a). Consistent with this hypothesis, aPKC aurA double mutants show strong suppression of the aurA supernumerary neuroblast phenotype, consistent with aPKC functioning downstream from AurA. While it is possible that loss of aPKC suppresses the phenotype in a nonspecific way (e.g., by arresting neuroblast cell proliferation or inducing neuroblast apoptosis), we fail to observe similarly strong suppression of the brat supernumerary neuroblast phenotype in $a$ PKC brat double mutants (Lee et al. 2006b). This shows that aPKC functions more specifically in the AurA pathway than in the Brat pathway.

The only other detectable cortical polarity defect seen in aurA mutant neuroblasts is a delocalization of Numb from the basal cortex. A similar Numb defect is seen during asymmetric cell division of pupal SOPs in aurA mutants (Fig. 4K; Berdnik and Knoblich 2002), perhaps reflecting a specific and direct regulation of Numb by AurA, although Numb is not phosphorylated by AurA in vitro (Berdnik and Knoblich 2002). The importance of the Numb delocalization phenotype is revealed by the ability of Numb overexpression in neuroblasts to rescue most of the aurA mutant phenotype (all except the com- 
Lee et al.
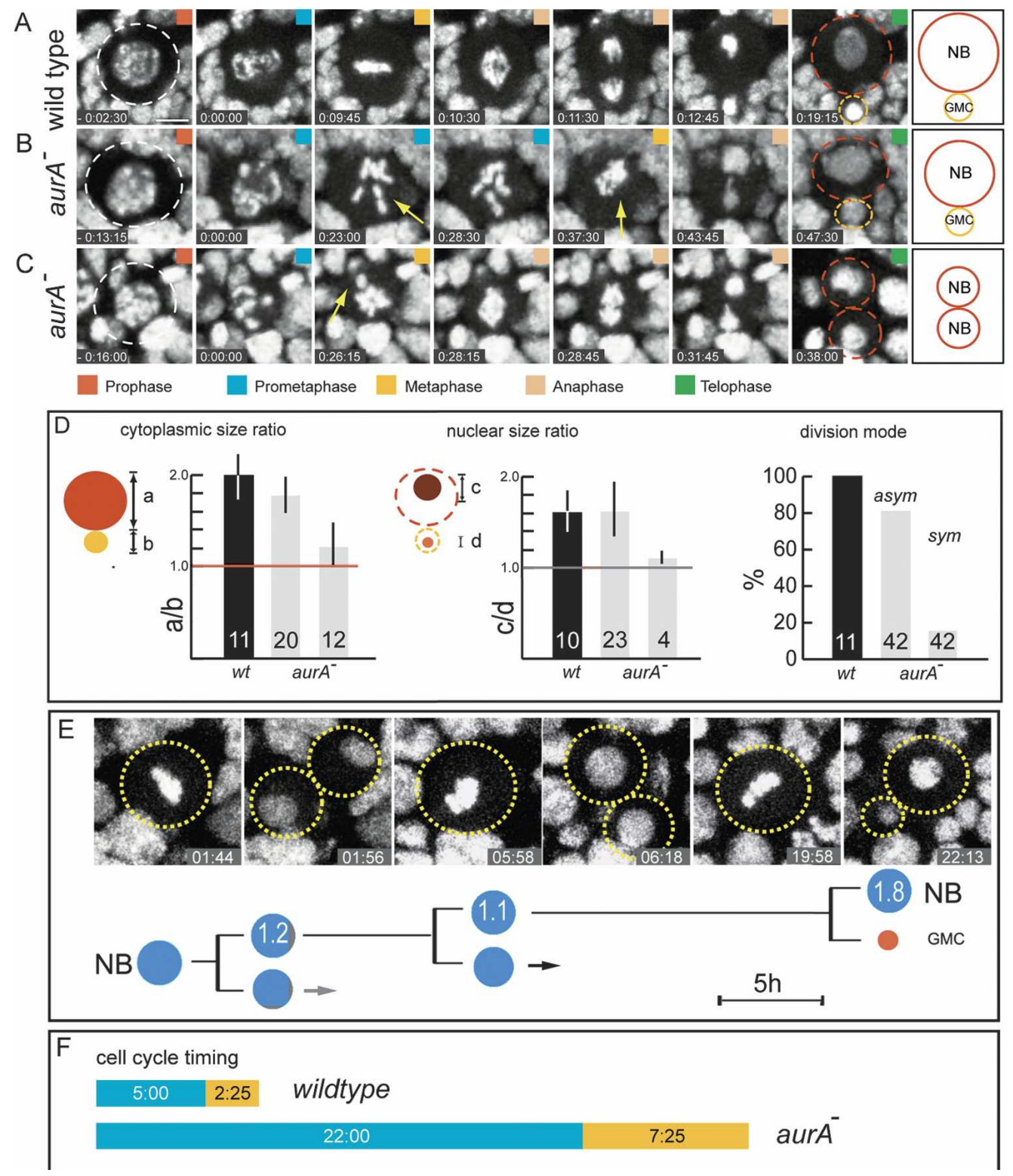

Figure 5. aurora- $A$ neuroblasts undergo symmetric cell division to expand the neuroblast population. $(A-D)$ aur $A$ mutant neuroblasts (NB) can divide symmetrically. (A) Wild-type neuroblasts divide asymmetrically. $(B)$ The majority of aurA mutant neuroblasts divide asymmetrically. $(C)$ A minority of aurA mutant neuroblasts undergo symmetric cell division. Bar, $5 \mu$ m. $(D)$ Quantification of neuroblast sibling cell size and nuclear size in wild type and aurA mutants. Data are derived from movies of wild-type and aur $A^{14641} /$ aur $A^{17961}$ neuroblasts expressing histone:GFP (His2AvD:GFP) under its native promoter (Clarkson and Saint 1999). Neuroblasts and GMCs are outlined in the first and last panel. 0:00:00 (hours:minutes:seconds) indicates nuclear envelope breakdown; colored boxes indicate cell cycle stages. (E) Example of an aurA neuroblast lineage tree. The neuroblast divides twice symmetrically and then undergoes a fully asymmetric cell division. White numbers are the ratio of sibling cell diameters; $1.0-1.25$, symmetric; $>1.5$, asymmetric. (Gray arrow) Neuroblast initiated division in the last frame of the movie; (black arrow) neuroblast remained in interphase. (F) aurA mutants have delays in cell cycle progression. aurA neuroblasts dividing symmetrically or asymmetrically have similar delays and are pooled for analysis. (Blue) Prometaphase; (yellow) metaphase. Numbers are in minutes:seconds (mean values); $n \geq 11$ for each genotype.

ponent due to spindle orientation defects; see below). Thus, Numb acts downstream from AurA to inhibit neuroblast self-renewal. Numb joins Mira/Pros/Brat as proteins that are partitioned into the GMC during neuroblast asymmetric cell division, where they function to inhibit neuroblast self-renewal (Fig. 6; Bello et al. 2006; Betschinger et al. 2006; Lee et al. 2006b).

Where does AurA function to inhibit neuroblast selfrenewal? AurA appears to be required in the neuroblast lineage, and not in surrounding glial cells or nonneuro- 


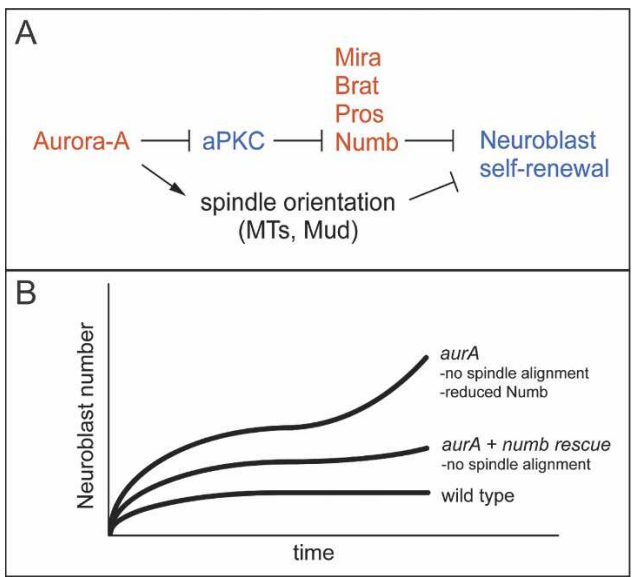

Figure 6. AurA inhibits neuroblast self-renewal. (A) AurA inhibits aPKC basal localization and promotes Numb basal localization; Numb inhibits neuroblast self-renewal. AurA independently promotes spindle-cortex alignment. Although $a P K C$ mutants affect all known basal proteins (Numb, Mira, Brat, and Pros), aurA mutants only show detectable changes in Numb localization. (B) Schematic illustrating the consequences of losing one or both AurA pathways for inhibiting neuroblast selfrenewal. (Top line) aurA mutants have defects in both basal Numb localization and spindle alignment; loss of both pathways leads to massive increases in neuroblast numbers. (Middle line) aurA mutants overexpressing Numb have defects only in the spindle alignment pathway and have a smaller increase in neuroblast numbers (comparable to mud or cnn mutants) (Fig. 3C; Siller et al. 2006; Bowman et al. 2006). (Bottom line) Wildtype neuroblast numbers. Time axis depicts 0-120 h ALH.

nal tissues of the larva, because neuroblast-specific expression of either AurA or the downstream component Numb can rescue most of the aurA supernumerary neuroblast phenotype. This shows that AurA is not required outside the neuroblast lineage to inhibit neuroblast selfrenewal. Within the neuroblast, AurA appears to function in the cytoplasm and not at the centrosome, because cnn mutants lack all detectable AurA centrosomal localization yet do not match the aurA supernumerary neuroblast phenotype. We conclude that AurA acts in the neuroblast cytoplasm to promote aPKC/Numb cortical polarity and spindle-to-cortex alignment.

How does Numb inhibit neuroblast self-renewal in the GMC? Numb is a well-characterized inhibitor of Notch signaling that is segregated into the GMC (Frise et al. 1996; Guo et al. 1996; Zhong et al. 1996; O'Connor-Giles and Skeath 2003; Hutterer and Knoblich 2005), and Notch signaling is active in larval neuroblasts but not in GMCs (Almeida and Bray 2005). Thus the most obvious model is that Numb blocks Notch receptor signaling in the GMC. However, Notch mutant clones generated in larval neuroblasts do not affect neuroblast survival or clone size (Almeida and Bray 2005). Similarly, we have seen no change in neuroblast number in two different Notch-ts mutants (although we observed the expected small wing imaginal disc phenotype) (C.-Y. Lee, unpubl.). In addition, we did not observe supernumerary neuroblasts in larval neuroblast clones overexpressing the constitutively active Notch intracellular domain (C.-Y. Lee, unpubl.), although the same Notch intracellular domain generates the expected sibling neuron phenotype when expressed in the embryonic CNS (J. Boone and K. Robinson, unpubl.). Thus, Notch is an excellent candidate for promoting neuroblast self-renewal, but additional experiments will be needed to test this model more rigorously. In this context, it is interesting to note that Notch promotes stem cell self-renewal in mammals (Androutsellis-Theotokis et al. 2006; Fan et al. 2006).

We show that aurA mutant neuroblasts have essentially random orientation of the mitotic spindle relative to the apical/basal cortical polarity axis, resulting in a some neuroblasts dividing symmetrically (in size and cortical polarity markers). This phenotype may arise due to lack of astral microtubule interactions with the neuroblast cortex, as aurA mutant neuroblasts have reduced astral microtubule length (Giet et al. 2002). Alternatively, AurA may affect spindle orientation by phosphorylating proteins required for spindle orientation, such as Cnn, Pins, or Mud. For example, Mud has a consensus AurA/Ipl1 phosphorylation site within its microtubule-binding domain, and it will be interesting to determine if this site needs to be phosphorylated for Mud to bind microtubules. Spindle orientation defects only generate part of the supernumerary neuroblast phenotype in aurA mutant brains, however, because overexpression of Numb can rescue most of the phenotype without rescuing spindle alignment, and cnn or mud mutants have nearly random spindle alignment but only a modest increase in neuroblast number (Fig. 3C; Bowman et al. 2006). Thus, we propose that spindle orientation defects and cortical polarity defects combine to generate the dramatic supernumerary neuroblast phenotype seen in aurA mutants (Fig. 6).

Mammalian aurA has been termed an oncogene due to its overexpression in several cancers, its ability to promote proliferation in certain cell lines, and the fact that reduced levels lead to multiple centrosomes, mitotic delay, and apoptosis (for review, see Giet et al. 2005). However, an in vivo aurA mutant phenotype has not yet been reported. In contrast, we find that aurA loss-of-function mutations result in a neuroblast "brain tumor" phenotype, including prolonged neuroblast proliferation during pupal stages when wild-type neuroblasts have stopped proliferating. aurA mutants do not, however, have the imaginal disc epithelial overgrowth seen in other Drosophila tumor suppressor mutants (for review, see Hariharan and Bilder 2006), and aurA mutant neuroblasts have a delay in cell cycle progression. We propose that the aurA supernumerary neuroblast phenotype is not due to loss of growth control or a faster cell cycle time, but rather due to a cell fate transformation from a differentiating cell type (GMC) to a proliferating cell type (neuroblast).

We conclude that AurA restrains neuroblast numbers using two pathways: first by promoting Numb localization into the GMC, and second by promoting alignment of the mitotic spindle with the cortical polarity axis. Absence of the first pathway leads to increased neuro- 
blasts at the expense of GMCs, whereas absence of the second pathway leads to increased neuroblasts due to symmetric cell division. It will be interesting to determine whether mammalian AurA uses one or both pathways to regulate stem cell asymmetric division and selfrenewal.

\section{Materials and methods}

Fly stocks and genetics

All mutant chromosomes were balanced over $C y O$, actin::GFP or TM3 actin:: GFP, Ser, e. We used Oregon R as wild type, and the following mutant chromosomes:

aPKC ${ }^{\mathrm{k} 06403}$ (Rolls et al. 2003)

aurA ${ }^{8839}$ (this work)

$\operatorname{aurA}^{14641}$ (this work)

aurA ${ }^{17961}$ (this work)

aurA ${ }^{87 \mathrm{Ac}-3}$ (Bloomington stock 6188)

FRT82B aurA ${ }^{14641}$ (this work)

UAS-aurA:GFP (this work)

UAS-numb (Knoblich et al. 1997)

worniu-Gal4 (Albertson and Doe 2003; Lee et al. 2006a)

$\mathrm{cnn}^{\text {HK21 }}$ (Megraw et al. 1999)

Df(3R)T-61 (Bloomington stock 3003)

elav-Gal4(C155), UAS-mCD8:GFP, hsp70-FLP; tubulin-Gal80,

FRT40A (Lee and Luo 1999)

elav-Gal4(C155), UAS-mCD8:GFP, hsp70-FLP; FRT82B tubu-

lin-Gal80 (Lee and Luo 1999)

w; FRT82B, mira ${ }^{z z 178}$ (Caussinus and Gonzalez 2005)

w; brat ${ }^{150}$, FRT40A (Betschinger et al. 2006)

w; numb ${ }^{2}$, FRT40A (this work)

w; FRT82B pros $^{17}$ (Reddy and Rodrigues 1999)

His2AvD:GFP (Clarkson and Saint 1999)

hs-flp(F38) (Bloomington stock 5258)

actin-FRT-Draf+-FRT-nuc.lacZ (Struhl and Basler 1993)

actin-FRT-stop-FRT-Gal4

UAS-Notch-intra (Struhl and Greenwald 2001)

Notch-ts1 (Bloomington stock 2533; chromosome cleaned of background mutations and provided by Ross Cagan, Washington University, St. Louis, MO|

Notch-ts2 (Bloomington stock 3075)

All clone experiments (MARCM, actin-Gal4, or actinnuc.lacZ) were done using standard methods (Lee and Luo 2001; Lee et al. 2006b) by giving the larval progeny a $1-\mathrm{h} 37^{\circ} \mathrm{C}$ heat shock at $24 \mathrm{~h} \mathrm{ALH}$, development at $25^{\circ} \mathrm{C}$, and clone analysis at $96 \mathrm{~h} \mathrm{ALH}$

\section{Antibodies and imaging}

Larvae brains were dissected in Schneider's medium (Sigma), fixed in $100 \mathrm{mM}$ Pipes $(\mathrm{pH}$ 6.9), $1 \mathrm{mM}$ EGTA, 0.3\% Triton $\mathrm{X}-100$, and $1 \mathrm{mM} \mathrm{MgSO}$ containing 4\% formaldehyde for 23 min and blocked for $1 \mathrm{~h}$ in $1 \times$ PBS containing $1 \%$ BSA and $0.3 \%$ Triton X-100 (PBS-BT) supplemented with 0.01 M glycine and $0.1 \%$ goat normal serum. After blocking, specimens were washed in PBS-BT for $1 \mathrm{~h}$ and incubated with primary antibodies in PBS-BT overnight at $4^{\circ} \mathrm{C}$. Brains were stained as described previously (Albertson and Doe 2003; Lee et al. 2006a). Pupal nota were dissected and stained as described previously (Manning and Doe 1999). We used guinea pig anti-Mira (1:400), rat anti-Mira (1:500), guinea pig anti-Numb (1:1000; J. Skeath), rat anti-Brat (1:100), mouse anti-Pros MR1A bioreactor supernatant (1:100), rabbit anti-aPKÇ (1:1000; Santa Cruz Biotechnology), rabbit anti-Goi (1:1000), mouse anti-Insc (1:1000; Bill Chia), rat anti-Pins (1:500), rat anti-Par6 (1:50), guinea pig anti-Baz (1:400), rabbit anti-Scribble (1:2500), mouse anti-Discs large (1:100; Developmental Study Hybridoma Bank [DSHB]), rabbit anti-Mud (1:1000), guinea pig anti-Dpn (1:1), mouse anti-Elav (1:50; DSHB), rat anti-Elav (1:10; DSHB), mouse anti-BrdU (1:50,

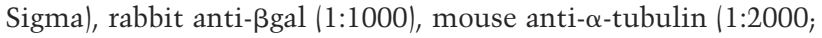
Sigma), Rat anti- $\alpha$-tubulin (1:100; Serotec), mouse anti- $\gamma$-tubulin (1:2000; Sigma), rabbit anti-AurA (1:200; J. Knoblich), rabbit anti-phosphohistoneH3 (1:1000; Upstate Biotechnology), rabbit anti-Cnn (1:1000; T. Kaufman), rabbit anti-GFP (1:1000; Torrey Pine), mouse anti-CD8 (1:100; Caltag), mouse anti-Hindsight "concentrated" (1:100; DHSB), and secondary antibodies from Molecular Probes. Antibodies without named sources were made in the laboratory; details are available on request.

\section{BrdU pulse/chase experiments}

Larvae were fed with BrdU (1 mg/mL; Roche) in media for $4 \mathrm{~h}$, and then one pool was processed for BrdU staining (pulse experiments) and a second pool was grown without BrdU for $24 \mathrm{~h}$ before fixation and BrdU staining (pulse/chase experiments). Larval brains were dissected, processed, and antibody-stained as described above with the exception that larval brains were treated in $2 \mathrm{~N} \mathrm{HCl}$ for $30 \mathrm{~min}$ prior to primary anti-BrdU antibody staining.

\section{Neuroblast counting and brain orientation}

A larval brain lobe consists of the medially localized central brain and the laterally localized optic lobe. Neuroblasts can be unambiguously identified by expression of Wor, Dpn, and Mira and the absence of the neuronal/glial differentiation markers Elav and Repo (Albertson and Doe 2003; Lee et al. 2006a). Central brain neuroblasts (the focus of this study) can be distinguished from optic lobe neuroblasts due to their medial-superficial location in the brain, larger size, and dispersed pattern (optic lobe neuroblasts laterally positioned in the brain and spaced very closely to each other, forming a ribbon that flanks and encircles the highly stereotypical epithelial-shaped optic lobe cells) (Lee et al. 2006a). All images of neuroblasts shown were collected from central brain,; all brains were mounted with dorsal surface up and ventral surface down, and the midline is to the left in all panels.

\section{Time-lapse imaging of larval neuroblasts}

aur $A^{14641}$ was recombined with His2AvD:GFP /Clarkson and Saint 1999) and crossed to aur $A^{17961}$. Late second and/or early third instar aurA mutant larvae were picked based on the lack of the dominant marker Tubby and dissected as previously described (Siller et al. 2005, 2006). Larvae were dissected and mounted in D-22 media (US Biological) with $1 \%$ bovine growth serum (BGS; HyClone) and imaged on a Bio-Rad Radiance 2000 confocal or Leica SP2 microscope using a $60 \times 1$.4NA oil-immersion objective. Time-lapse sequences were processed using ImageJ and Imaris 5.0.1, 64-bit (Bitplane).

\section{Acknowledgments}

We thank James Skeath, Juergen Knoblich, Cayenato Gonzalez, Bill Chia, Jordan Raff, Fumio Matsuzaki, Carl Thummel, Ross Cagan, and the Bloomington stock center for fly stocks and/or antibody reagents, and Janet Hanawalt for administrative assistance. C.-Y.L. was supported by a Damon Runynon post-doc- 
toral fellowship and a Career Award in Biomedical Research from the Burroughs Wellcome Fund, A.B. was supported by an NIH grant (GM73670), and C.Q.D. was supported by the Howard Hughes Medical Institute, where he is an Investigator.

\section{References}

Albertson, R. and Doe, C.Q. 2003. Dlg, Scrib and Lgl regulate neuroblast cell size and mitotic spindle asymmetry. Nat. Cell Biol. 5: 166-170.

Almeida, M.S. and Bray, S.J. 2005. Regulation of post-embryonic neuroblasts by Drosophila Grainyhead. Mech. Dev. 122: 1282-1293.

Androutsellis-Theotokis, A., Leker, R.R., Soldner, F., Hoeppner, D.J., Ravin, R., Poser, S.W., Rueger, M.A., Bae, S.K., Kittappa, R., and McKay, R.D. 2006. Notch signalling regulates stem cell numbers in vitro and in vivo. Nature 442: 823-826.

Bello, B., Reichert, H., and Hirth, F. 2006. The brain tumor gene negatively regulates neural progenitor cell proliferation in the larval central brain of Drosophila. Development 133: 2639-2648.

Berdnik, D. and Knoblich, J.A. 2002. Drosophila Aurora-A is required for centrosome maturation and actin-dependent asymmetric protein localization during mitosis. Curr. Biol. 12: 640-647.

Betschinger, J. and Knoblich, J.A. 2004. Dare to be different: Asymmetric cell division in Drosophila, C. elegans and vertebrates. Curr. Biol. 14: R674-R685.

Betschinger, J., Mechtler, K., and Knoblich, J.A. 2006. Asymmetric segregation of the tumor suppressor brat regulates self-renewal in Drosophila neural stem cells. Cell 124: 12411253.

Bowman, S.K., Neumuller, R.A., Novatchkova, M., Du, Q., and Knoblich, J.A. 2006. The Drosophila NuMA Homolog Mud regulates spindle orientation in asymmetric cell division. Dev. Cell 10: 731-742.

Broadus, J. and Doe, C.Q. 1997. Extrinsic cues, intrinsic cues and microfilaments regulate asymmetric protein localization in Drosophila neuroblasts. Curr. Biol. 7: 827-835.

Cai, Y., Yu, F., Lin, S., Chia, W., and Yang, X. 2003. Apical complex genes control mitotic spindle geometry and relative size of daughter cells in Drosophila neuroblast and pI asymmetric divisions. Cell 112: 51-62.

Caussinus, E. and Gonzalez, C. 2005. Induction of tumor growth by altered stem-cell asymmetric division in Drosophila melanogaster. Nat. Genet. 37: 1125-1129.

Cheetham, G.M., Knegtel, R.M., Coll, J.T., Renwick, S.B., Swenson, L., Weber, P., Lippke, J.A., and Austen, D.A. 2002. Crystal structure of aurora-2, an oncogenic serine/threonine kinase. J. Biol. Chem. 277: 42419-42422.

Clarkson, M. and Saint, R. 1999. A His2AvDGFP fusion gene complements a lethal His2AvD mutant allele and provides an in vivo marker for Drosophila chromosome behavior. DNA Cell Biol. 18: 457-462.

$\mathrm{Du}, \mathrm{J}$. and Hannon, G.J. 2004. Suppression of p160ROCK bypasses cell cycle arrest after Aurora-A/STK15 depletion. Proc. Nat1. Acad. Sci. 101: 8975-8980.

Dutertre, S., Descamps, S., and Prigent, C. 2002. On the role of aurora-A in centrosome function. Oncogene 21: 6175-6183.

Fan, X., Matsui, W., Khaki, L., Stearns, D., Chun, J., Li, Y.M., and Eberhart, C.G. 2006. Notch pathway inhibition depletes stem-like cells and blocks engraftment in embryonal brain tumors. Cancer Res. 66: 7445-7452.

Farris, S.M., Robinson, G.E., Davis, R.L., and Fahrbach, S.E. 1999. Larval and pupal development of the mushroom bod- ies in the honey bee, Apis mellifera. J. Comp. Neurol. 414: 97-113.

Fichelson, P. and Gho, M. 2004. Mother-daughter precursor cell fate transformation after Cdc2 down-regulation in the Drosophila bristle lineage. Dev. Biol. 276: 367-377.

Frise, E., Knoblich, J.A., Younger-Shepherd, S., Jan, L.Y., and Jan, Y.N. 1996. The Drosophila Numb protein inhibits signaling of the Notch receptor during cell-cell interaction in sensory organ lineage. Proc. Nat1. Acad. Sci. 93: 11925-11932.

Fuse, N., Hisata, K., Katzen, A.L., and Matsuzaki, F. 2003. Heterotrimeric G proteins regulate daughter cell size asymmetry in Drosophila neuroblast divisions. Curr. Biol. 13: 947954.

Giet, R., McLean, D., Descamps, S., Lee, M.J., Raff, J.W., Prigent, C., and Glover, D.M. 2002. Drosophila Aurora A kinase is required to localize D-TACC to centrosomes and to regulate astral microtubules. J. Cell Biol. 156: 437-451.

Giet, R., Petretti, C., and Prigent, C. 2005. Aurora kinases, aneuploidy and cancer, a coincidence or a real link? Trends Cell Biol. 15: 241-250.

Glover, D.M., Leibowitz, M.H., McLean, D.A., and Parry, H. 1995. Mutations in aurora prevent centrosome separation leading to the formation of monopolar spindles. Cell 81: 95105

Guo, M., Jan, L.Y., and Jan, Y.N. 1996. Control of daughter cell fates during asymmetric division: Interaction of Numb and Notch. Neuron 17: 27-41.

Hariharan, I.K. and Bilder, D. 2006. Regulation of imaginal disc growth by tumor-suppressor genes in Drosophila. Annu. Rev. Genet. 40: 335-361.

Hutterer, A. and Knoblich, J.A. 2005. Numb and $\alpha$-Adaptin regulate Sanpodo endocytosis to specify cell fate in Drosophila external sensory organs. EMBO Rep. 6: 836-842.

Izumi, Y., Ohta, N., Hisata, K., Raabe, T., and Matsuzaki, F. 2006. Drosophila Pins-binding protein Mud regulates spindle-polarity coupling and centrosome organization. Nat. Cell Biol. 8: 586-593.

Knoblich, J.A., Jan, L.Y., and Jan, Y.N. 1997. Asymmetric segregation of the Drosophila numb protein during mitosis: Facts and speculations. Cold Spring Harb. Symp. Quant. Biol. 62: 71-77.

Lee, T. and Luo, L. 1999. Mosaic analysis with a repressible cell marker for studies of gene function in neuronal morphogenesis. Neuron 22: 451-461.

Lee, T. and Luo, L. 2001. Mosaic analysis with a repressible cell marker (MARCM) for Drosophila neural development. Trends Neurosci. 24: 251-254.

Lee, C.Y., Robinson, K.J., and Doe, C.Q. 2006a. Lgl, Pins and aPKC regulate neuroblast self-renewal versus differentiation. Nature 439: 594-598.

Lee, C.Y., Wilkinson, B.D., Siegrist, S.E., Wharton, R.P., and Doe, C.Q. 2006b. Brat is a Miranda cargo protein that promotes neuronal differentiation and inhibits neuroblast selfrenewal. Dev. Cell 10: 441-449.

Manning, L. and Doe, C.Q. 1999. Prospero distinguishes sibling cell fate without asymmetric localization in the Drosophila adult external sense organ lineage. Development 126: 2063 2071.

Megraw, T.L., Li, K., Kao, L.R., and Kaufman, T.C. 1999. The centrosomin protein is required for centrosome assembly and function during cleavage in Drosophila. Development 126: 2829-2839.

Morrison, S.J. and Kimble, J. 2006. Asymmetric and symmetric stem-cell divisions in development and cancer. Nature 441: 1068-1074.

Nordlander, R.H. and Edwards, J.S. 1970. Postembryonic brain 
Lee et al.

development in the Monarch butterfly, Dancus plexippis plexippus, L. III. Morphogenesis of centers other than the optic lobe. Wilhelm Roux Arch. Entw Mech. Org. 164: 247260.

Nowakowski, J., Cronin, C.N., McRee, D.E., Knuth, M.W., Nelson, C.G., Pavletich, N.P., Rogers, J., Sang, B.C., Scheibe, D.N., Swanson, R.V., et al. 2002. Structures of the cancerrelated Aurora-A, FAK, and EphA2 protein kinases from nanovolume crystallography. Structure 10: 1659-1667.

O'Connor-Giles, K.M. and Skeath, J.B. 2003. Numb inhibits membrane localization of Sanpodo, a four-pass transmembrane protein, to promote asymmetric divisions in Drosophila. Dev. Cell 5: 231-243.

Prokop, A. and Technau, G.M. 1994. Normal function of the mushroom body defect gene of Drosophila is required for the regulation of the number and proliferation of neuroblasts. Dev. Biol. 161: 321-337.

Reddy, G.V. and Rodrigues, V. 1999. Sibling cell fate in the Drosophila adult external sense organ lineage is specified by prospero function, which is regulated by Numb and Notch. Development 126: 2083-2092.

Rolls, M.M., Albertson, R., Shih, H.P., Lee, C.Y., and Doe, C.Q. 2003. Drosophila aPKC regulates cell polarity and cell proliferation in neuroblasts and epithelia. J. Cell Biol. 163: 1089-1098.

Siegrist, S.E. and Doe, C.Q. 2005. Microtubule-induced Pins/ Gai cortical polarity in Drosophila neuroblasts. Cell 123: 1323-1335.

Siller, K.H., Serr, M., Steward, R., Hays, T.S., and Doe, C.Q. 2005. Live imaging of Drosophila brain neuroblasts reveals a role for Lis1/dynactin in spindle assembly and mitotic checkpoint control. Mol. Biol. Cell 16: 5127-5140.

Siller, K.H., Cabernard, C., and Doe, C.Q. 2006. The NuMArelated Mud protein binds Pins and regulates spindle orientation in Drosophila neuroblasts. Nat. Cell Biol. 8: 594-600.

Spana, E.P. and Doe, C.Q. 1995. The prospero transcription factor is asymmetrically localized to the cell cortex during neuroblast mitosis in Drosophila. Development 121: 31873195.

Struhl, G. and Basler, K. 1993. Organizing activity of wingless protein in Drosophila. Cell 72: 527-540.

Struhl, G. and Greenwald, I. 2001. Presenilin-mediated transmembrane cleavage is required for Notch signal transduction in Drosophila. Proc. Natl. Acad. Sci. 98: 229-234.

Yu, F., Cai, Y., Kaushik, R., Yang, X., and Chia, W. 2003. Distinct roles of $\mathrm{G} \alpha \mathrm{i}$ and $\mathrm{G} \beta 13 \mathrm{~F}$ subunits of the heterotrimeric G protein complex in the mediation of Drosophila neuroblast asymmetric divisions. J. Cell Biol. 162: 623-633.

Zhong, W., Feder, J.N., Jiang, M.M., Jan, L.Y., and Jan, Y.N. 1996. Asymmetric localization of a mammalian numb homolog during mouse cortical neurogenesis. Neuron 17: 4353. 


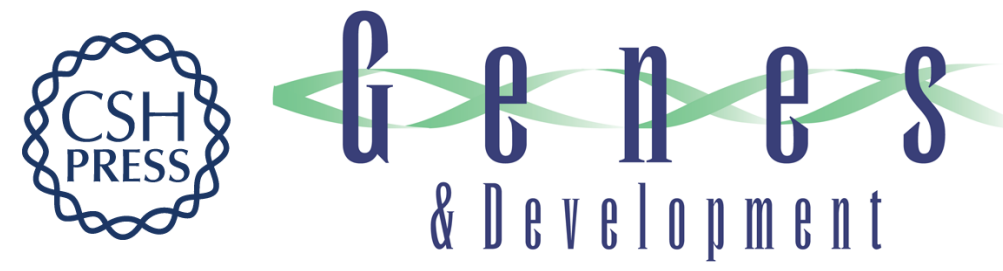

\section{Drosophila Aurora-A kinase inhibits neuroblast self-renewal by regulating aPKC/Numb cortical polarity and spindle orientation}

Cheng-Yu Lee, Ryan O. Andersen, Clemens Cabernard, et al.

Genes Dev. 2006, 20:

Access the most recent version at doi:10.1101/gad.1489406

Supplemental
Material http://genesdev.cshlp.org/content/suppl/2006/12/20/20.24.3464.DC1

References This article cites 49 articles, 15 of which can be accessed free at:

http://genesdev.cshlp.org/content/20/24/3464.full.html\#ref-list-1

License

Email Alerting Receive free email alerts when new articles cite this article - sign up in the box at the top

Service

right corner of the article or click here.

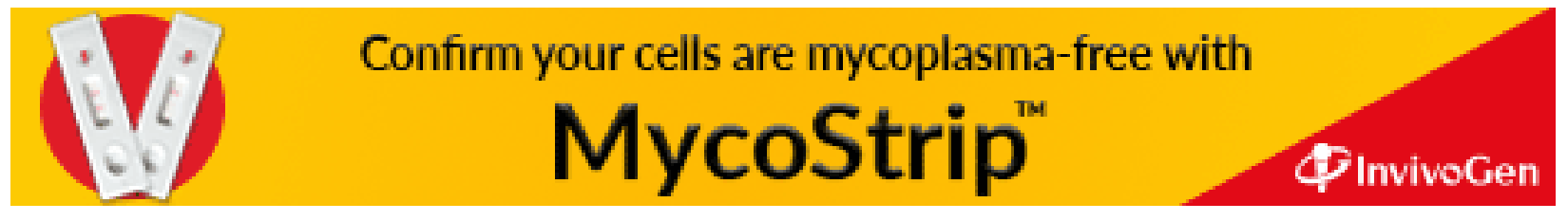

